\title{
A flexible, three material, 3D-printed, shear force sensor for use on finger tips
}

\author{
Gerjan Wolterink $^{1,2}$, Remco Sanders ${ }^{1}$, Gijs Krijnen ${ }^{1}$ \\ ${ }^{1}$ Robotics And Mechatronics group, University of Twente, Enschede, The Netherlands \\ ${ }^{2}$ Biomedical Signal and Systems, University of Twente, Enschede, The Netherlands \\ Email: gerjan.wolterink@utwente.nl
}

\begin{abstract}
This paper shows the development and characterization of a 3D-printed flexible finger tip sensor that measures both shear and normal forces. The sensor is fabricated using three material fused deposition modelling (FDM) 3D-printing. The sensing principle is based on the mechanical deformation of the finger tips caused by normal and shear-forces. Therefore, the sensor is flexible and can measure the interaction forces between the environment and the finger tips, while keeping the loss of touch sensation low. Characterization shows the sensor is capable of distinguishing between normal and a shear-force components.
\end{abstract}

Keywords - 3D-Printing, Biomedical, Flexible, Soft, Shearforce sensor

\section{INTRODUCTION}

The interaction forces between the fingertips and the external environment are of great value in for example quantitative assessment of the rehabilitation of the upper extremities. Typical forces at the finger tips range up to $10 \mathrm{~N}$ for shear and $50 \mathrm{~N}$ for normal forces with a preferable high sensitivity below $10 \mathrm{~N}$ [1]-[3]. The interaction forces between the fingertips and the external environment can potentially be obtained by placing force sensors on the fingertips. However, current force sensors have shown two main difficulties. Firstly, the sensors are usually made of stiff materials leading to the loss of touch sensation by the user. Secondly, current sensors do not enable good sensor to skin attachment making the sensor more sensitive to unwanted movements caused by the external forces [4]. Additive manufacturing, or 3Dprinting, can overcome these difficulties due to design freedom, customisability and availability of soft materials. With the advent of multi-material printers, conductive and dielectric materials can be printed in one go. This enables the creation of personalised sensing structures, making for a useful tool for prosthetic assistive devices and soft-robotic applications. In this work, we show the development of flexible 3D-printed shear-force sensors using multi-material fused deposition modelling (FDM) printing technology. The first force sensors made

This works was developed within the SoftPro project, funded by the European Union's Horizon 2020 Research and Innovation Programme under Grant Agreement No. 688857 using this technology were based on piezoresistive and capacitive principles [5]-[8]. Another sensing principle is based on the anisotropy of 3D-printed carbon doped thermoplastic polyurethane (TPU) [9].

The goal of this work is to create 3 degrees of freedom (DoF) force sensors, which include 2 shear-force components, that measure the interaction forces between the environment and the finger tips, with minimal loss of touch sensation of the user, by using the mechanical deformation of the finger tips themselves. A comparable concept, using traditional fabrication methods, that uses the finger deformation of a robotic finger is demonstrated by Yin et al. [2]. However, this sensor is limited in measuring the shear-force in two directions and is insensitive to normal forces. Furthermore, the design is made for robotic sensing and therefore does not facilitate touch sensation of the underlying finger.

\section{SENSOR PRINCIPLE}

\section{A. Sensor Geometry}

The sensing principle of the finger tip sensor is based on the mechanical deformation of the fingertip. Fig. 1 represents a simplified model of the finger tip with circumferential sensor structure. When a normal or a shear-force is applied to the finger tip, the soft tissue moves relative to the stiff nail-bed and finger bone. If a flexible band is placed around the tip of the finger and mounted on the nail, a strain is induced in the sides of the band. By placing strain sensors at these locations, the finger deformation can be measured. A normal force exerted on the finger will result in a compression of both strain gauges. Whereas a shear-force in one direction leads to a compression on one side and an elongation on the other side.
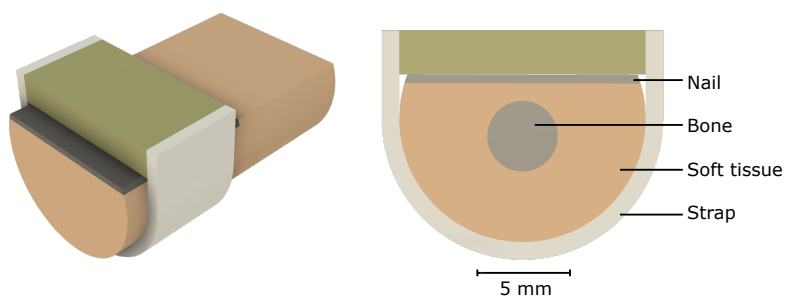

Fig. 1. Finger model with strap, left: 3D-model, right: cross-section 


\section{B. Finger Model}

To simulate the movement of the finger tissue due to an applied normal or shear-force, a finite element method (FEM) simulation is performed using Fusion360 (Autodesk, USA). Fig. 1 shows the model of the finger with surrounded elastic strap. The mechanical properties of the finger bone and nail are assumed to be equal to steel. The surrounding finger tissue had a Young's modulus of $34 \mathrm{kPa}$ and Poisson ratio of 0.3 [10]. The strap around the finger is simulated to be an elastic TPU material mounted on a rigid plastic block above the finger nail, with a Young's modulus of 12 and $2240 \mathrm{MPa}$ and a Poisson ratio of 0.49 and 0.38 for the strap and rigid block, respectively. Fig. 2 shows the modelled strain for a $5 \mathrm{~N}$ normal force and a $5 \mathrm{~N}$ shear-force in combination with a $1 \mathrm{~N}$ normal force. It can be clearly seen that the strap deforms due to the deformation of the soft tissue of the finger.

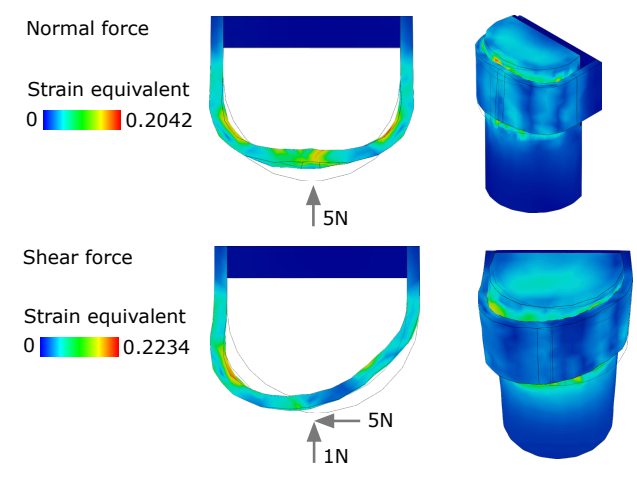

Fig. 2. Modelled strain equivalent due to a normal and shear-force.

\section{Methodology}

\section{A. Sensor design and Fabrication}

Fig. 3 shows the design and the dimensions of the sensor. The gray material of the strap is a non-conductive thermoplastic polyurethane (TPU) (NinjaFlex, Fenner Drives, USA), the strain gauges (black parts) are made of carbon doped conductive TPU (PI-ETPU, Palmiga Innovation, Sweden). The blue part is printed from polylactic acid (PLA) (3D-Prima, Sweden), the holes on top of this part are for mounting the sensor in the test setup. The sensor is printed in one go using a Diabase H-Series Hybrid (Diabase Engineering, USA) FDM 3D-Printer. The layer height and infill are set in slicer software (Simplify3D, Inc., USA) to be $150 \mu \mathrm{m}$ and $100 \%$. The resistance of the strain gauges is in the range of $15 \mathrm{k} \Omega$.

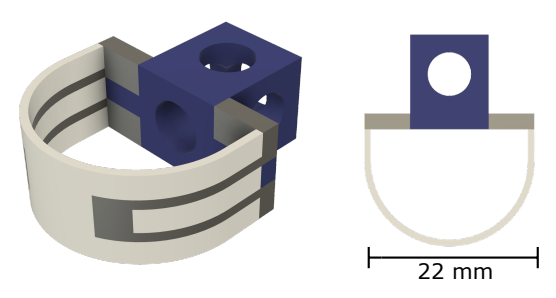

Fig. 3. CAD model of the sensor, left: 3D-model, right: cross-section

\section{B. Measurement set-up}

The resistance change of the strain gauges is read out using a voltage divider setup. One side of each strain gauge is connected to a dc-voltage of $1 \mathrm{~V}$, the other side is connected to an oscilloscope (PicoScope 5443B, Pico Technology, UK) and a $47 \mathrm{k} \Omega$ series resistor to the ground, see Fig. 4. Connection to the sensor is made by melting in a stranded wire to the connection pads at the top. The used input voltage is $1 \mathrm{~V}$ and is provided by the signal generator of the oscilloscope.

To study the normal force the sensor is mounted in line with a force-controlled linear actuator (SMAC LCA25-050-15F, SMAC, USA) and pressed against a vertical aluminium plate covered with a polyimide film. The shear-force is measured in another configuration. Here the sensor is placed in a $90^{\circ}$ angle on the actuator and dragged over a horizontal platform covered with 80-grit sandpaper. The normal force in the shearforce measurements is controlled by moving the platform up and down. A lab scale (E0D120, Ohaus, USA) underneath the platform provides the normal force information. Both oscilloscope and linear actuator are controlled using Matlab (Mathworks Inc., USA). To simulate the presence of a finger tip a finger phantom was 3D-printed and the sensor was mounted around it. The stiff nail and bone part are printed in solid infill using PLA and the soft compressible finger tissue is simulated by TPU printed at $30 \%$ infill, see Fig. 5 .
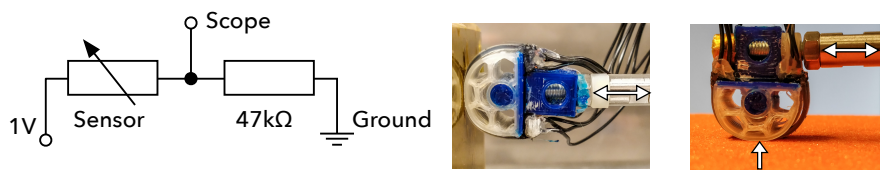

Fig. 4. The resistance change of one strain gauge is of strain gauge is read out using the the metal rod of the linear actuator illustrated voltage divider setup. in the measurement setup for normal (left) and shear-forces (right). Arrows indicate the direction of the forces.

\section{Measurement protocol}

In the normal force setup the sensor is preloaded with $5.5 \mathrm{~N}$. Next a sinusoidal force is applied with an amplitude of $5 \mathrm{~N}$ for $10 \mathrm{~s}$. The sinusoidal force is applied at $5 \mathrm{~Hz}$. In the shear-force setup a constant normal force of $5 \mathrm{~N}$ is applied. The applied shear-force is a $1 \mathrm{~Hz}$ sinusoidal force with an amplitude of $5 \mathrm{~N}$. The oscilloscope data is recorded at a sample frequency of $192 \mathrm{kHz}$. During the analysis this data is low-pass filtered using a 4th order Butterworth filter with corner frequency of $100 \mathrm{~Hz}$. Drift is removed using a high-pass $3 \mathrm{rd}$ order Butterworth filter with corner frequency of $0.5 \mathrm{~Hz}$. To see the shear-force direction, the signals of sensor 1 are subtracted from the signals of sensor 2 .

\section{RESULTS}

\section{A. Normal force}

Fig. 6 shows the resistance change of the filtered data of sensor 1 and 2, caused by a sinusoidal normal force of 3 
periods. It can be seen that the sensitivities of the sensors are not completely equal. Therefore, the difference of the signals of sensors 1 and 2 is not completely 0 over time. Fig. 7 shows the resistance change against the force obtained from 5 times a $10 \mathrm{~N}$ load cycle. To show the drift, this data is only low-pass filtered.

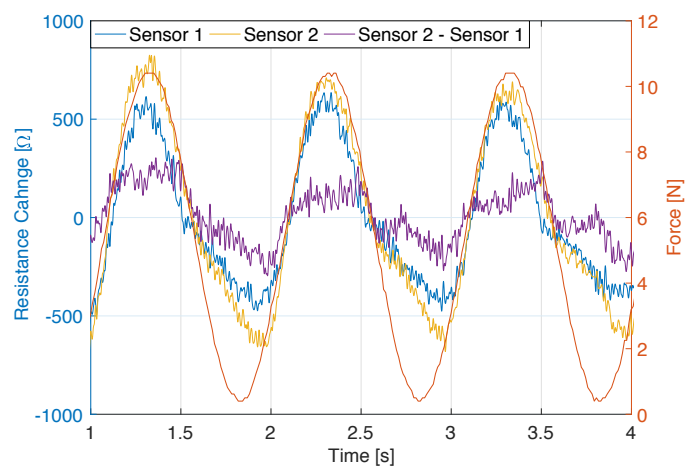

Fig. 6. Bandpass filtered data showing the resistance change of sensor 1 and 2 induced by a normal force.
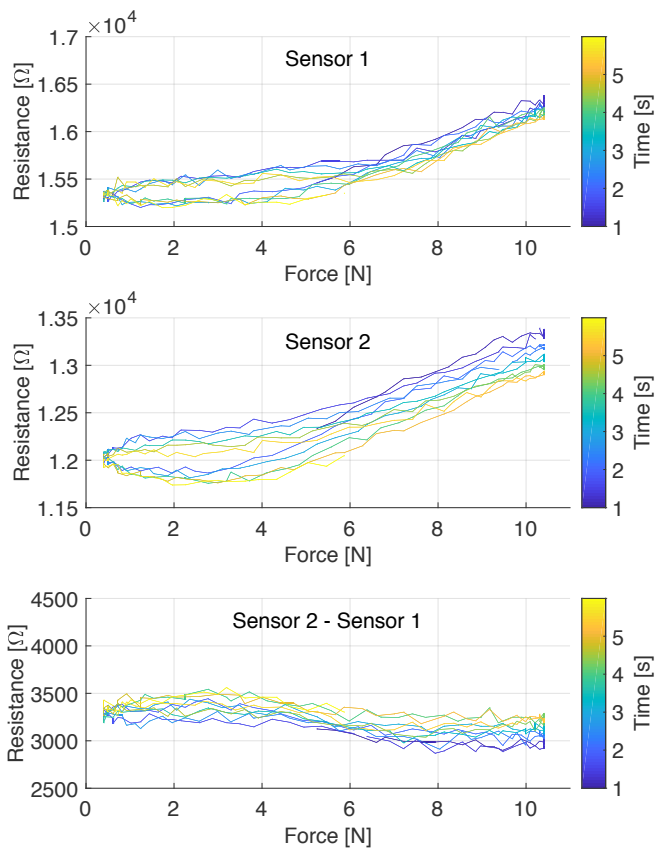

Fig. 7. Resistance change against the applied normal force for 5 load cycles. The data is only low-pass filtered

\section{B. Shear-force}

Fig. 8 shows the resistance change of the filtered data of sensor 1 and 2, due to a changing shear-force over 3 periods. It can be seen that sensor 1 is more sensitive in one direction with respect to sensor 2 and visa versa. However, the difference of the resistance changes of sensor 2 and 1 shows a sinusoidal wave clearly correlated to the applied shear-force as shown in Fig. 8 .

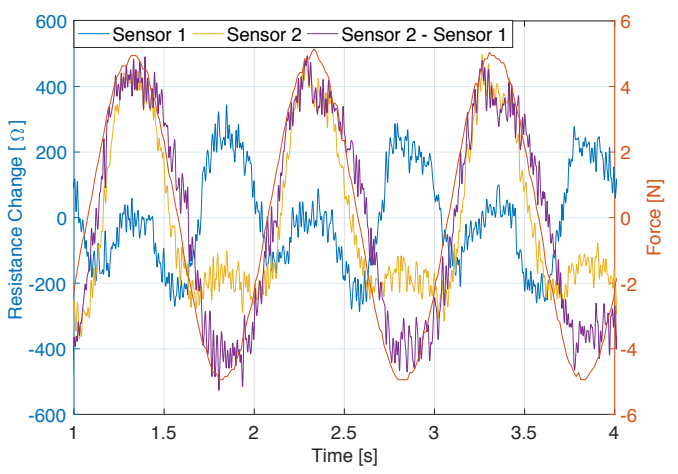

Fig. 8. Bandpass filtered data showing the resistance change of sensor 1 and 2 induced by an applied shear-force and a constant $5 \mathrm{~N}$ normal force.

\section{DISCUSSION}

There seems to be a small delay of around $40 \mathrm{~ms}$ between the applied force and sensor readout (Fig. 8). It is unclear if this is caused by mechanical properties of the sensor or by communication delays between the measurement hardware. Future improvements of the read out methods could provide information about these communications delays, to compensate for this delay in the signal analysis.

The sensitivity of both sensors is not equal, this can be caused by imperfection of the printing process or by misalignments in the measurement set-up. Fig. 8 shows that the sensitivity ratio between compression and elongation is lower for sensor 1 relative to sensor 2. Furthermore, Fig. 7 shows that both sensors are less sensitive to normal forces in the $1 \mathrm{~N}$ to $5 \mathrm{~N}$ range than in the $5 \mathrm{~N}$ to $10 \mathrm{~N}$ range.

In this work the normal and shear-forces are applied separately, the next step would be to apply both forces simultaneously. Since both elongation and compression cause resistance changes, further understanding of the mechanical deformation of the fingertip is needed. This can help to develop algorithms that can separate the normal and shear-force and their directions.

Design improvements can be made by increasing the sensitivity of the sensor, e.g. by increasing the length of the strain gauges by adding extra meanderings of the conductive material. Further design improvements can be made to decrease the impact of the sensor on the user. Since the sensors are at the side of the finger, large parts of the strap material can be removed from around the finger tip, increasing the touch sensation area for the user.

\section{CONCLUSiOnS}

We have demonstrated a working concept of a 3D-Printed flexible finger sensor that can measure both shear and normal forces by using the mechanical deformation of a phantom finger tip. Since the sensors are on the side of the finger, the fingertip only has to be minimally covered. To fully exploit the functionality of the sensor, more advanced algorithms for signal analysis need to be made to separate shear from normal force. 


\section{REFERENCES}

[1] R. A. Brookhuis, H. Droogendijk, M. J. de Boer, R. G. Sanders, T. S. Lammerink, R. J. Wiegerink, and G. J. Krijnen, "Six-axis force-torque sensor with a large range for biomechanical applications," Journal of micromechanics and microengineering, vol. 24, no. 3, p. 035015, 2014.

[2] J. Yin, V. J. Santos, and J. D. Posner, "Bioinspired flexible microfluidic shear force sensor skin," Sensors and Actuators A: Physical, vol. 264, pp. 289-297, 2017.

[3] E. Battaglia, M. Bianchi, A. Altobelli, G. Grioli, M. G. Catalano, A. Serio, M. Santello, and A. Bicchi, "Thimblesense: a fingertipwearable tactile sensor for grasp analysis," IEEE transactions on haptics, vol. 9, no. 1, pp. 121-133, 2015.

[4] H. Kortier, H. Schepers, and P. Veltink, "Identification of object dynamics using hand worn motion and force sensors," Sensors, vol. 16, no. 12, p. $2005,2016$.

[5] M. Schouten, R. Sanders, and G. Krijnen, "3D printed flexible capacitive force sensor with a simple micro-controller based readout," in 2017 IEEE SENSORS, Oct 2017, pp. 1-3.

[6] A. Dijkshoorn, P. Werkman, M. Welleweerd, G. Wolterink, B. Eijking, J. Delamare, R. Sanders, and G. J. Krijnen, "Embedded sensing: integrating sensors in 3-d printed structures," Journal of Sensors and Sensor Systems, vol. 7, no. 1, p. 169, 2018.

[7] S. J. Leigh, R. J. Bradley, C. P. Purssell, D. R. Billson, and D. A. Hutchins, "A simple, low-cost conductive composite material for $3 \mathrm{~d}$ printing of electronic sensors," PloS one, vol. 7, no. 11, p. e49365, 2012.

[8] M. B. Kirkpatrick, J. A. Tarbutton, T. Le, and C. Lee, "Characterization of $3 \mathrm{~d}$ printed piezoelectric sensors: Determiniation of d 33 piezoelectric coefficient for 3d printed polyvinylidene fluoride sensors," in SENSORS, 2016 IEEE. IEEE, 2016, pp. 1-3.

[9] G. Wolterink, R. Sanders, and G. Krijnen, "Thin, flexible, capacitive force sensors based on anisotropy in 3d-printed structures," in 2018 IEEE SENSORS. IEEE, 2018, pp. 1-4.

[10] S. Shimawaki and N. Sakai, "Quasi-static deformation analysis of a human finger using a three-dimensional finite element model constructed from ct images," Journal of Environment and Engineering, vol. 2, no. 1, pp. 56-63, 2007. 